\title{
Características de la canal y de la carne en la raza caprina Bermeya. Comparación con el Ternasco de Aragón y lechales de la raza Murciano-Granadina
}

\author{
A. Guerrero', J.S. Lemes ${ }^{1,2}$, M.M. Campo ${ }^{1}$, J.L. Olleta ${ }^{1}$, E. Muela ${ }^{1}$, \\ V.C. Resconi' ${ }^{1}$, V.M. Guerra ${ }^{3}$, F. Assis-Macedo ${ }^{4}$ y C. Sañudo ${ }^{1, *}$ \\ 1 Departamento Producción Animal y Ciencia de los Alimentos, Universidad de Zaragoza, Instituto \\ Agroalimentario (IA2), C/ Miguel Servet 177, 50013 Zaragoza, España \\ 2 Departamento Produção Animal, Universidade Federal de Santa Maria, Av. Independência, $n^{\circ} 3751$, \\ Bairro Vista Alegre, 98300-000 - Palmeira das Missões - RS. Brasil \\ 3 Asociación de Criadores de cabra Bermeya, ACRIBER, España, Caserío Gromaz, La Borbolla, 33598 \\ Llanes, Asturias, España \\ 4 Programa de Pós Graduação em Zootecnia, Universidade Federal de Sergipe, São Cristovão, 49100- \\ 000 - SE. Brasil
}

\begin{abstract}
Resumen
Se ha estudiado la calidad de la raza caprina Bermeya: lechales ligeros (BL) y cabritos pesados de explotación tradicional (BP), que se compararon con productos reconocidos (lechal de la raza Murciano-Granadina (MG) y corderos amparados bajo la IGP Ternasco de Aragón (TA). Los animales fueron criados y sacrificados en condiciones comerciales, y seleccionados por las respectivas Asociaciones de Productores y Consejo Regulador. Los porcentajes de hueso y músculo fueron mayores, y el de grasa inferior, en Bermeya $(P<0,001)$, encontrándose, igualmente, diferencias en la composición química de la carne. Los animales bermeyos presentaron valores de $\mathrm{pH}$ superiores $(P<0,001)$ a los de MG o TA. Los BP presentaron un índice de rojo y croma mayor respecto a BL. Tanto el tipo de animal como el tiempo de maduración afectaron significativamente a la textura. Así, MG mostró el mayor valor al $20 \%$ de compresión (C20) a 2 días de maduración $(P<0,001)$ y el TA los menores al $80 \%$ de compresión (C80) en todos los tiempos de maduración $(P<0,001)$. Se observaron diferencias significativas en casi todos los ácidos grasos analizados y en la intensidad de olor a especie $(P<0,05)$, terneza, jugosidad e intensidad de flavor a especie $(P<0,001)$, valoradas por un panel entrenado. En aceptabilidad global y de la terneza (consumidores), $\mathrm{BL}$ presentaron puntuaciones superiores $(P<0,001)$ a BP, aunque inferiores a las de los productos de referencia. Los resultados obtenidos sugieren, no obstante, una buena aceptación de los productos de la raza Bermeya y un cierto grado de diferenciación con los productos con los que han sido comparados.
\end{abstract}

Palabras clave: Cordero ligero, calidad carne, ácidos grasos, perfil sensorial, consumidores.

\section{Abstract \\ Carcass and meat characteristics from commercial products of Bermeya goat breed. A comparative study in relation with Ternasco de Aragón light lamb and Murciano-Granadina kids}

Meat quality characteristics from Bermeya goat breed were studied. Light suckling kids $(n=12)$ and heavy kids from the traditional production system $(n=10)$ were compared with reference products from the

* Autor para correspondencia: csanudo@unizar.es

http://dx.doi.org/10.12706/itea.2016.017 
Spanish market (suckling kids from Murciano-Granadina breed (MG); $n=12$ and light lamb included in the Ternasco de Aragón Protected Geographic Indication (TA); $n=12$ ). Animals were reared and slaughtered under commercial conditions, and selected from the respective Breeder Associations. Bermeya carcasses showed greater percentage of bone and muscle $(P<0.001)$, and lower percentage of fat in comparison with the other types, differing also in the chemical meat composition. $\mathrm{pH}$ values were higher in both Bermeya breed types than in MG and TA. Redness and Chroma increased with age in the Bermeya breed. Both type of animal and ageing affected textural variables. Murciano-Granadina meat showed the highest value of compression at $20 \%(C 20)$ at 2 days of ageing $(P<0.001)$ and TA the smallest at $80 \%$ of compression rate $(C 80)$ at all the ageing times analyzed $(P<0.001)$. Differences between commercial types were observed both in most fatty acids and in the intensity of species odor $(P<0.05)$, tenderness, juiciness and specie flavour $(P<0.001)$ evaluated by trained panelists. A higher tenderness and overall acceptability scores were given by consumers to light Bermeya in comparison with heavy Bermeya kids $(P<0.001)$, and both were lower than those of the reference products. Results suggest a good acceptability and certain degree of differentiation from Bermeya breed products with respect to those compared with.

Key words: Light lamb, meat quality, fatty acids, sensory profile, consumers.

\section{Introducción}

La raza caprina Bermeya es autóctona de Asturias, constituyendo hasta mediados del siglo XX la población caprina más importante de la rama occidental de la Cornisa Cantábrica española (Figueroa et al., 2003). Actualmente cuenta con un censo de unas 3.000 cabezas (Asociación de Criadores de la raza Bermeya-ACRIBER), localizadas fundamentalmente en su comunidad de origen (Asturias), formando parte del grupo de razas en peligro de extinción del Catálogo Oficial de Razas de Ganado de España (BOE, 2009).

La aptitud de la cabra Bermeya es doble (carne y leche), siendo la leche un producto secundario con el que se elaboran diversos quesos tradicionales (Cabrales o Gamonedo) fundamentalmente en los rebaños del área de influencia del Parque Nacional de los Picos de Europa (Figueroa et al., 2003). Esta raza, a pesar de encontrarse bien adaptada y haber demostrado una excepcional rusticidad al criarse en regiones montañosas de condiciones orográficas difíciles, se ve afectada en su conservación y expansión por diversos factores humanos y sociales, fundamentalmente debidos al reducido número de explotaciones existentes, a la dimensión de las mismas, así como a la competencia que sufre por parte de otros genotipos existentes en la zona (Figueroa et al., 2003). Los sistemas de producción utilizados con la raza Bermeya son de tipo tradicional, en los que el ganado caprino tiene un claro papel ecológico-medio ambiental, por su labor de limpieza del monte cuando las densidades de pastoreo son las adecuadas y de conservación de la biodiversidad (García et al., 2012). La cría tradicional de cabritos de raza Bermeya está basada en pastoreo extensivo donde los animales son sacrificados o bien como lechales, a la edad aproximada de 3-4 meses con un peso canal de unos 8-10 kg, o más pesados, a la edad de 5-10 meses, castrados y con pesos de canal de 10-12 kg hasta 15-18 $\mathrm{kg}$, en función de la alimentación disponible en los pastos, de las peculiaridades de cada ganadero y de la demanda puntual de su carne para la hostelería (Esteban, 2008).

Actualmente, el desarrollo y demanda por parte del consumidor de productos locales (ya sean quesos o carne), bajo el amparo de distintas marcas regionales, están aumentando, así como el valor económico agregado de dichos productos. Por tanto, el desarrollo de marcas de calidad en carne fresca reclama la 
existencia de trabajos en los que se tipifiquen dichos productos, analizando su posible variabilidad y calidad, en comparación con otros ya consolidados en el mercado (Martínez-Cerezo et al., 2005; Bonvillani et al., 2010).

Por todo esto, el objetivo fundamental de este trabajo ha sido el estudio de la calidad de la canal y de la carne de los dos tipos comerciales principales existentes en la raza Bermeya (lechal ligero y animales de pasto pesados), comparando estos productos con otros que están reconocidos dentro del mercado nacional de carne de los pequeños rumiantes; como serían lechales de la raza Murciano-Granadina, raza caprina más extendida en España (Zurita-Herrera et al., 2011), o el "Ternasco de Aragón", producto ovino cuyo reconocimiento de calidad se encuentra amparado bajo una Indicación Geográfica Protegida. Con todo ello, este trabajo puede sentar las bases de la calidad de la carne que permitan dar, si es posible, a los productos locales un valor añadido, manteniendo la diversificación de la producción caprina en Asturias y, en definitiva, aumentar las posibilidades de conservación de la raza Bermeya.

\section{Material y métodos}

\section{Animales y diseño experimental}

Se utilizaron 46 animales, machos, pertenecientes a las razas caprinas Bermeya $(n=22)$ y Murciano Granadina $(n=12)$, así como a la raza ovina Rasa Aragonesa $(n=12)$ dentro de la Indicación Geográfica Protegida Ternasco de Aragón. Todos los animales fueron criados en condiciones comerciales y fueron seleccionados, para ser representativos de su correspondiente tipo de producto según los estándares locales y el correspondiente pliego de condiciones en el caso del tipo ovino, por personal técnico de la Asociación correspondiente: ACRIBER, CAPRIGRAN (Asociación de
Criadores de raza Murciano-Granadina, Granada) y Grupo Pastores en el caso del Ternasco de Aragón.

La cabra de raza Bermeya es un animal tremendamente rústico y adaptado a las condiciones orográficas, nada fáciles, del Principado de Asturias. Estas condiciones determinan en gran medida su manejo siendo, en la mayoría de los casos, rebaños que pasan la mayor parte del año en completa libertad por los diferentes montes de la región, a excepción del periodo de tiempo comprendido entre diciembre y marzo. Si bien últimamente existe un mayor control de los animales debido a la presencia de un elevado número de lobos, lo que en ocasiones ha conducido en cambios en el manejo de los animales encerrándolos todos los días o trasladándolos a pastos más cercanos a las explotaciones donde la presencia del lobo es menos abundante. En cualquier caso, en muy raras ocasiones se suministra a las madres complemento en forma de pienso, siendo más frecuente el suministro de paja. Durante el invierno se produce la paridera y se amamantan los cabritos antes de salir sus madres a pastar y cuando éstas vuelven al atardecer a las explotaciones.

Los animales comerciales de raza Bermeya fueron sacrificados con dos pesos-edades y sistemas de producción diferentes: 12 de ellos pertenecían al tipo lechal (Bermeya ligera, $\mathrm{BL})$, siendo sacrificados con 2,5-3 meses de edad, y 10 animales castrados del tipo Bermeya pesada (BP) sacrificados con 8-10 meses (Tabla 1). Los cabritos bermeyos fueron alimentados con leche materna y, en el caso de los de más edad, pastos vecinales de los municipios situados en el este de Asturias, en la comarca de Cangas de Onís, dentro de la zona sur de influencia de los Picos de Europa (Esteban, 2008; Álvarez, 2008). En el presente estudio, en ningún momento se suministró a los animales pienso o complemento alguno, de acuerdo al manejo y costumbres tradicionales en origen. 
Tabla 1. Características de la canal, pH y composición química del músculo Longissimus thoracis de cabritos comerciales de raza Bermeya sacrificados a dos pesos, respecto a dos tipos comerciales de referencia ${ }^{1}$

Table 1. Carcass characteristics, $\mathrm{pH}$ and chemical composition from Longissimus thoracis muscle of commercial Bermeya kids slaughtered at light (BL) and heavy weight (BP), and two commercial small ruminant types

\begin{tabular}{|c|c|c|c|c|c|}
\hline & $\mathrm{BL}$ & BP & MG & TA & Sig. \\
\hline Peso canal fría, kg & $7,01 \pm 1,86 b$ & $10,52 \pm 1,50 a$ & $5,64 \pm 0,71 c$ & $10,79 \pm 0,64 a$ & *** \\
\hline Longitud externa canal, cm & $44,68 \pm 2,81 b$ & $53,05 \pm 3,02 a$ & $39,80 \pm 1,37 c$ & $52,00 \pm 1,35 a$ & $* * *$ \\
\hline Compacidad canal ${ }^{2}, \mathrm{~kg} / \mathrm{cm}$ & $0,15 \pm 0,33 c$ & $0,19 \pm 0,02 b$ & $0,14 \pm 0,15 d$ & $0,21 \pm 0,01 \mathrm{a}$ & $* * *$ \\
\hline \multicolumn{6}{|l|}{ Composición tisular $3 \%$} \\
\hline Músculo & $69,71 \pm 1,72 a$ & $69,17 \pm 2,52 \mathrm{a}$ & $64,62 \pm 1,94 b$ & $62,65 \pm 1,82 c$ & $* * *$ \\
\hline Grasa subcutánea & $1,04 \pm 0,71 b$ & $2,26 \pm 1,91 b$ & $2,14 \pm 0,91 b$ & $5,68 \pm 1,74 a$ & $* * *$ \\
\hline Grasa intermuscular & $4,27 \pm 2,12 b$ & $4,53 \pm 2,08 b$ & $10,57 \pm 1,95 a$ & $9,72 \pm 1,34 a$ & $* * *$ \\
\hline Grasa total & $5,31 \pm 2,75 c$ & $6,80 \pm 3,51 c$ & $12,71 \pm 2,59 b$ & $15,40 \pm 1,88 a$ & $* * *$ \\
\hline Hueso & $24,99 \pm 1,77 a$ & $24,03 \pm 1,83 a b$ & $22,68 \pm 1,60 \mathrm{bc}$ & $21,96 \pm 1,33 c$ & $* * *$ \\
\hline $\mathrm{pH} 48 \mathrm{~h}$ & $5,98 \pm 0,30 a$ & $5,95 \pm 0,14 a$ & $5,67 \pm 0,16 b$ & $5,59 \pm 0,03 b$ & $* * *$ \\
\hline \multicolumn{6}{|l|}{ Composición química, \% } \\
\hline Humedad & $77,86 \pm 0,88 a$ & $75,76 \pm 1,94 b$ & $75,45 \pm 1,14 b$ & $75,65 \pm 1,77 b$ & $* * *$ \\
\hline Proteína & $17,14 \pm 2.11 b$ & $18,86 \pm 1,43 a$ & $18,84 \pm 1,64 a$ & $17,30 \pm 0,66 b$ & $* *$ \\
\hline Grasa & $1,92 \pm 0,80 b$ & $2,80 \pm 1,88 b$ & $7,49 \pm 4,06 a$ & $3,62 \pm 1,86 b$ & $* * *$ \\
\hline Cenizas & $1,06 \pm 0,11 b$ & $1,11 \pm 0,07 b$ & $1,37 \pm 0,13 a$ & $1,04 \pm 0,04 b$ & $* * *$ \\
\hline
\end{tabular}

${ }^{1}$ BL: Bermeya ligera, BP: Bermeya pesada, MG: Murciano-Granadina (lechal, ligera), TA: Ternasco de Aragón IGP (ovino).

2 Peso canal fría/longitud externa canal.

3 Procedente de la disección de la espalda izquierda.

Distintas letras en una fila indican diferencias $(P<0,05)$.

Como producto de referencia, para las canales ligeras se utilizaron 12 lechales de lactancia natural de la raza Murciano-Granadina, así como 12 corderos de raza Rasa Aragonesa, amparados bajo la IGP "Ternasco de Aragón", presentando un peso de canal similar al del cabrito tradicional bermeyo y criados de acuerdo al pliego de condiciones prescrito en dicha Indicación Geográfica Protegida, incluyendo pienso y paja a voluntad hasta el sacrificio (MAGRAMA, 2014). Cuando los animales alcanzaron sus correspondientes pesos comerciales, fueron sacrificados en mataderos homologados por la Unión Europea próximos a las explotaciones de origen, faenándose siguiendo la normativa correspondiente sobre protección de animales en el momento del sacrificio. El sacrificio de los animales de raza Bermeya se realizó en Margolles, Asturias, el de la Murciano-Granadina en Granada y el del "Ternasco de Aragón" en Zaragoza. 
Muestreo y análisis laboratoriales

A las 24 horas post-sacrificio todas las canales fueron transportadas en condiciones de refrigeración al laboratorio de la Facultad de Veterinaria de Zaragoza, donde se obtuvo el peso canal fría. Sobre la canal se midió su longitud externa, con la que se calculó su índice de compacidad (peso canal fría/longitud canal).

De cada animal se retiró la espalda izquierda, siguiendo la metodología descrita por Colomer et al. (1988). Estas se envasaron al vacío y se conservaron en congelación $\left(-18^{\circ} \mathrm{C}\right)$ hasta su disección, con la que se evaluó la composición porcentual de músculo, grasa subcutánea, grasa intermuscular, hueso y otros (grandes vasos, tendones y el ganglio pre-escapular).

Además, se extrajeron ambos músculos Longissimus thoracis y lumborum (LTL), midiéndose el $\mathrm{pH}$ en la zona craneal mediante un pHmétro portátil CRISON provisto de un electrodo de penetración.

La distribución de muestras para los posteriores análisis laboratoriales fue la siguiente: la porción torácica del LTL derecho se destinó para los análisis de textura (T3-T13) y el resto (T13-L6), para realizar el estudio de consumidores. La porción torácica del LTL izquierdo se destinó para el análisis de ácidos grasos y composición química, y el resto para análisis sensorial mediante panel entrenado. Todas las muestras fueron envasadas al vacío. Las muestras para el análisis de ácidos grasos y composición química se congelaron inmediatamente, y el resto fueron refrigeradas a $4{ }^{\circ} \mathrm{C}$ hasta que completaron el tiempo de maduración post-sacrificio correspondiente (4 días para los análisis sensoriales, panel entrenado y consumidores, y 2, 4, 8 y 12 días en las respectivas sub-muestras utilizadas para evaluar la textura), momento en el que fueron congeladas $\left(-18^{\circ} \mathrm{C}\right)$ hasta su análisis posterior.

La composición química fue realizada de acuerdo a los siguientes métodos oficiales: humedad (ISO-R-1442. ISO, 1997), proteína (ISO-R-937 ISO, 1978), grasa (ISO-R-1443. ISO, 1973) y cenizas (ISO-R-936. ISO, 1998).

El color se evaluó en el corte realizado en el LTL izquierdo a la altura de la T13. Tras diez minutos de exposición al oxígeno, se midió con un colorímetro CR-200 (Minolta, ángulo de visión $2^{\circ}$, iluminante D65), realizando tres medidas por muestra, con las que se determinaron las variables del sistema CIELab: luminosidad $\left(L^{*}\right)$, índice de rojo $\left(a^{*}\right)$ e índice de amarillo $\left(b^{*}\right)$, calculándose posteriormente el tono $\left(\mathrm{H}^{\circ}\right)$ y croma $\left(\mathrm{C}^{*}\right)$ de la carne.

La textura se valoró mediante la utilización del equipo Instron 4301 (Instron Limited Corporation, High Wycombe, Reino Unido), en el que se instaló una célula de compresión (Lepetit y Culioli, 1994). Para ello, tras la descongelación en condiciones de refrigeración, cada muestra fue dividida en prismas rectangulares de $1 \mathrm{~cm}^{2}$ de sección siguiendo el eje paralelo a la fibras musculares, evaluándose la fuerza necesaria $\left(\mathrm{N} / \mathrm{cm}^{2}\right)$ para comprimir la muestra el $20 \%$ o al $80 \%$ de dicha sección.

La extracción de la grasa intramuscular se realizó tras descongelación de acuerdo a la técnica de Bligh y Dyer (1959), seguido de una metilación con $\mathrm{KOH}$ en metanol. La identificación de los ácidos grasos se realizó mediante cromatografía de gases, utilizando un cromatógrafo HP 6890 de columna capilar SP $2380(100 \mathrm{~m} \times 0,25 \mathrm{~mm} \times 0,20 \mu \mathrm{m})$ y nitrógeno como gas portador (Carrillho et al., 2009).

\section{Análisis sensorial}

El análisis sensorial con panel entrenado se llevó a cabo en cabinas normalizadas con luz roja mediante un panel compuesto por nueve personas seleccionadas y entrenadas para realizar dichas evaluaciones (UNE-EN ISO 85862. 2009). Las muestras se descongelaron en condiciones de refrigeración durante 24 horas y posteriormente fueron cocinadas en un 
grill de doble placa precalentado a $200^{\circ} \mathrm{C}$, sin ningún tipo de aditivo, hasta alcanzar una temperatura interna de $70^{\circ} \mathrm{C}$. Una vez que las muestras fueron cortadas en prismas cúbicos de unos $2 \mathrm{~cm}^{2}$ de sección, etiquetadas y distribuidas, los panelistas evaluaron en cada una de ellas doce atributos (olor a especie, olor a grasa, terneza, jugosidad, fibrosidad, untuosidad, flavor a especie, flavor metálico, flavor grasa, flavor lácteo, flavor ácido y flavor picante), más una nota de aceptabilidad global, mediante una escala semiestructurada de $10 \mathrm{~cm}$ desde: nada intenso, tierno, jugoso o fibroso (según atributo) a extremadamente intenso, tierno, jugoso o fibroso, respectivamente. Estos descriptores fueron decididos por el propio panel en una sesión previa de entrenamiento con carne equivalente a la posteriormente utilizada en el estudio. El diseño fue equilibrado por bloques completos, constando de 4 sesiones, 3 platos por sesión y 4 muestras, una de cada tipo comercial, por plato.

El análisis de consumidores se realizó bajo condiciones controladas en sala con luz blan$\mathrm{ca}$, en 10 sesiones, en las que participaron un total de 100 personas de entre 25 y 74 años de edad: $45 \%$ de hombres y un $55 \%$ de mujeres; un $7 \%$ tenía menos de 25 años, el $46 \%$ entre 26 y 45 , el $36 \%$ entre 46 y 65 y el $11 \%$ tenía más de 65 años. Para la preparación culinaria de las muestras se siguió el procedimiento anteriormente descrito. Cada consumidor evaluó, en presentación individual, una muestra de cada uno de los cuatro tipos de animales. Para evitar posibles efectos de unas muestras sobre otras, éstas se evaluaron en distinto orden para cada consumidor (Macfie et al., 1989), siguiendo un diseño de bloques completos. Se evaluó en cada muestra la aceptabilidad de la terneza, del sabor, así como su aceptación general, mediante una escala hedónica estructurada de 9 puntos, que variaba desde 1 (me desagrada extremadamente) a 9 (me gusta extremadamente), en la que se eliminó el punto medio.
Análisis estadístico

Se utilizó el paquete estadístico SPSS v19.0 para ver el efecto del tipo de animal sobre las variables estudiadas mediante un análisis de varianza (GLM). En el análisis sensorial, un primer GLM fue realizado con el panelista como efecto fijo, además de la sesión y del tipo de animal, para analizar el consenso en las valoraciones. Posteriormente, se analizó el efecto tipo de animal en un segundo análisis además del plato (sesión). En el test de consumidores se utilizó un modelo mixto, donde además del tipo de animal como efecto fijo, se consideró al consumidor como aleatorio. En los análisis de textura, se utilizó un GLM considerando como efectos fijos tanto el tipo de animal como el tiempo de maduración, junto con su posible interacción en dichas variables. Cuando el tipo de animal fue un efecto significativo, las diferencias entre medias se evaluaron mediante el test de Duncan $(P<0,05)$. Los resultados se presentan como medias de cada grupo, así como sus desviaciones típicas.

\section{Resultados y discusión}

\section{Calidad de la canal}

La Tabla 1 refleja el peso canal e índice de compacidad de los diferentes tipos de animales. Como era esperable debido a las diferencias de peso vivo, edad, raza y especie, hubo diferencias $(P<0,001)$ en dichas variables, presentando los lechales de la raza caprina Murciano-Granadina los valores más bajos. A pesar de no encontrarse diferencias entre el peso canal de los cabritos bermeyos pesados y el Ternasco de Aragón, este último presentó un índice de compacidad superior $(P$ $<0,001)$. Las diversas medidas morfológicas tomadas (datos no mostrados) reflejan que las medidas longitudinales fueron superiores 
para la especie caprina, mientras que las medidas transversales fueron superiores para la especie ovina, como ya observaran Sañudo et al. (2012) al comparar varias razas caprinas de lechales y una ovina (Churra), implicando una mayor compacidad de la canal y de la pierna en las canales de cordero y, por lo tanto, una mejor conformación. Los cabritos bermeyos pesados presentaron valores superiores que los cabritos bermeyos ligeros, resultado esperable ya que la compacidad aumenta con la edad y el peso (Sañudo et al., 1993).

La Tabla 1 igualmente recoge los resultados sobre la composición tisular de la espalda, que sería representativa de la composición global de la canal (Vergara, 2005). Se encontraron diferencias $(P<0,001)$ entre los tipos de animales estudiados en todos los tejidos, siendo mayor los porcentajes de hueso y de músculo en la raza Bermeya e inferior el de grasa total, respecto a los dos tipos de referencia comerciales (MG y TA), lo que demuestra una aceptable vocación carnicera en dicha raza y las particularidades de deposición de la grasa en las razas caprinas, especialmente cuando la aptitud no es lechera y en sistemas no intensificados. Sin embargo, entre los animales bermeyos, las diferencias no fueron significativas, a pesar de las diferencias de edad. Al comparar con los animales de referencia, las diferencias pueden ser atribuibles a la mayor ingestión energética de los lechales de la raza Murciano-Granadina por la mayor disponibilidad de leche, y a que la especie caprina, en comparación con la ovina, presenta una menor cantidad de grasa en la canal, al depositar una mayor cantidad de este tejido en la región abdominal (Sen et al., 2004).

\section{Calidad instrumental de la carne}

En relación al $\mathrm{pH}$ final medido a las 48 horas (Tabla 1), ambos tipos de la raza Bermeya presentaron unos valores más altos $(P<0,001)$ que los otros dos grupos de referencia, siendo igualmente superiores al rango de $\mathrm{pH}$ descrito por Sañudo et al. (2012) entre cinco razas caprinas que osciló entre 5,76-5,84. En dicho trabajo, como en el presente, la animales de la especie ovina presentaron un $\mathrm{pH}$ normal, cercano al 5,5, lo que refuerza la idea de que la especie caprina es más susceptible al estrés (Sañudo, 2008). En la Tabla 1 se muestran los resultados de la composición química del músculo LTL. Se encontraron diferencias ( $P$ $<0,001)$ en el porcentaje de humedad y grasa, así como en el de proteína y cenizas $(P<$ $0,01)$. En relación a los dos tipos bermeyos, los animales pesados presentaron un porcentaje inferior de humedad y superior de proteína respecto a los ligeros. Al comparar estos resultados con los de los tipos de referencia, se observó que mostraron un porcentaje inferior de grasa y cenizas respecto a la Murciano-Granadina $y$, de hecho, igualmente inferior al descrito en otras razas caprinas con peso canal comparable, los cuales oscilaron entre el 3,1 y 5,7 (Dhanda et al., 2003; Sen et al., 2004). Santos et al. (2008), al comparar características de caprinos con ovinos sacrificados con grado de madurez similar, encontraron un mayor porcentaje de humedad y menor de grasa en la especie caprina respecto a la ovina, dato que exclusivamente concuerda con los del presente trabajo al comparar la humedad de la carne de raza Bermeya (ligera) con la del Ternasco de Aragón. Los lechales de Murciano-Granadina presentaron los mayores porcentajes de grasa, lo que estaría probablemente asociado a una abundante dieta láctea y manejo intensivo.

En relación al color, tal y como refleja la Tabla 2, los valores de luminosidad no fueron diferentes dentro de los dos tipos de animales de raza Bermeya, presentando una carne ligeramente más oscura que los cabritos de raza Murciano-Granadina $(P<0,001)$, lo cual podría estar en parte relacionado con sus superiores valores de $\mathrm{pH}$. El tono $\left(\mathrm{H}^{\circ}\right)$ tampoco varió entre ambos tipos, siendo los lechales murciano-granadinos los que presentaron un 
Tabla 2. Color de la carne de cabritos comerciales de raza Bermeya sacrificados a dos pesos, respecto a dos tipos comerciales de referencia ${ }^{1}$

Table 2. Meat colour of Bermeya commercial kids slaughtered at two different weights compared with two commercial small ruminant types

\begin{tabular}{lccccc}
\hline & $\mathrm{BL}$ & $\mathrm{BP}$ & $\mathrm{MG}$ & TA & Sig. \\
\hline Luminosidad $\left(\mathrm{L}^{*}\right)$ & $43,51 \pm 2,91 \mathrm{bc}$ & $43,21 \pm 2,91 \mathrm{c}$ & $52,82 \pm 3,48 \mathrm{a}$ & $45,77 \pm 0,96 \mathrm{~b}$ & $* * *$ \\
Índice de rojo $\left(\mathrm{a}^{*}\right)$ & $11,82 \pm 2,09 \mathrm{~b}$ & $16,19 \pm 1,94 \mathrm{a}$ & $10,53 \pm 1,63 \mathrm{~b}$ & $16,30 \pm 0,94 \mathrm{a}$ & $* * *$ \\
Índice de amarillo $\left(\mathrm{b}^{*}\right)$ & $4,09 \pm 1,43$ & $4,55 \pm 1,09$ & $4,93 \pm 0,71$ & $4,77 \pm 0,26$ & $\mathrm{~ns}$ \\
Croma $\left(\mathrm{C}^{*}\right)$ & $12,54 \pm 2,37 \mathrm{~b}$ & $16,82 \pm 2,14 \mathrm{a}$ & $11,68 \pm 1,40 \mathrm{~b}$ & $16,99 \pm 0,94 \mathrm{a}$ & $* * *$ \\
Tono $\left(\mathrm{H}^{\circ}\right)$ & $18,68 \pm 4,12 \mathrm{~b}$ & $15,56 \pm 2,04 \mathrm{~b}$ & $25,53 \pm 5,34 \mathrm{a}$ & $16,32 \pm 0,89 \mathrm{~b}$ & $* * *$ \\
\hline
\end{tabular}

${ }^{1}$ BL: Bermeya ligera, BP: Bermeya pesada, MG: Murciano-Granadina (lechal, ligera), TA: Ternasco de Aragón IGP (ovino).

Distintas letras en una fila indican diferencias $(P<0,05)$.

valor superior a los demás grupos $(P<0,001)$, coincidiendo con lo descrito en esta misma raza por Sañudo et al. (2012). Otros estudios sobre ganado caprino (Dhanda et al., 2003) han mostrado diferencias en las variables de color $(a *, b *)$ entre diferentes razas caprinas. Sin embargo, en el presente trabajo, el índice de rojo varió dependiendo del tipo de animal estudiado $(P<0,001)$, ya que los animales más jóvenes y que sólo se alimentaron con leche, ya sean de raza Bermeya (tipo ligero) o Murciano-Granadina, presentaron unos valores inferiores a los animales de raza Bermeya pesados y Ternasco de Aragón. Este hecho está ligado al sistema de alimentación, ya que a* se asocia con la disponibilidad del hierro, y por lo tanto con la presencia de mioglobina, siendo ésta baja en animales jóvenes y en dietas lecheras (Sañudo et al., 1997, 2012). No hubo diferencias en el índice de amarillo, cuyos valores fueron similares a los encontrados en otros estudios (Sañudo, 2008; Sañudo et al., 2012).

Tanto el tipo de animal como el tiempo de maduración afectaron a la textura de la carne medida por compresión $(P<0,001)$, y la interacción entre ambos factores afectó a la compresión al $20 \%(P<0,001)$. Tal y como se recoge en la Tabla 3, las medidas al $20 \%$ de la compresión, relacionadas con el componente miofibrilar, no fueron diferentes ni entre los dos tipos de animales bermeyos (ligeros y pesados), ni a lo largo de la maduración en estos dos tipos de la raza Bermeya. En relación a los tipos comerciales de referencia, los bermeyos presentaron unos resultados similares al Ternasco de Aragón y menores, sólo en la maduración a 2 días $(P<0,001)$, a los obtenidos en la raza Murciano-Granadina. En cualquier caso, estos resultados evidencian una posible maduración precoz en la carne de la raza Bermeya, o su limitada capacidad de ablandamiento después de dos días de maduración, independientemente de la edad de sacrificio, que debería ser estudiada con mayor profundidad, una vez considerado el posible efecto del $\mathrm{pH}$, superior en la carne de los animales bermeyos. En relación a los valores al $80 \%$ de compresión, que están más relacionados con el tejido conectivo (Campo et al., 2000), se observa como el tipo bermeyo pesado obtuvo valores inferiores que el ligero sólo durante los cuatro primeros días de maduración e intermedios a 
Tabla 3. Textura del músculo Longissimus thoracis a lo largo de la maduración en cabritos comerciales de raza Bermeya sacrificados a dos pesos, respecto a dos tipos comerciales de referencia ${ }^{1}$

Table 3. Texture of Longissimus thoracis muscle thought ageing of Bermeya commercial kids slaughtered at two different weights and compared with two commercial small ruminant types

\begin{tabular}{|c|c|c|c|c|c|}
\hline & BL & BP & MG & TA & Sig. \\
\hline \multicolumn{6}{|c|}{ Compresión al 20\% } \\
\hline $2 d$ & $7,27 \pm 2,41 b$ & $6,89 \pm 2,48 b$ & $16,14 \pm 4,32 a, x$ & $9,30 \pm 2,53 b, x$ & $* * *$ \\
\hline $4 d$ & $8,03 \pm 4,55$ & $5,99 \pm 2,87$ & $7,74 \pm 3,26$ y & $5,74 \pm 1,26 y$ & ns \\
\hline $8 d$ & $6,60 \pm 2,06$ & $6,73 \pm 1,46$ & $6,73 \pm 2,63 y$ & $5,52 \pm 1,00 y$ & ns \\
\hline $12 \mathrm{~d}$ & $7,43 \pm 3,27$ & $6,83 \pm 2,09$ & $6,76 \pm 2,84 y$ & $5,40 \pm 0,96 y$ & ns \\
\hline Sig. & ns & ns & $* * *$ & $* * *$ & \\
\hline \multicolumn{6}{|c|}{ Compresión al 80\% } \\
\hline $2 d$ & $63,94 \pm 14,87 a$ & $46,80 \pm 16,31 b, y$ & $57,72 \pm 15,59 a b, z$ & $31,67 \pm 8,17 c, y$ & $* * *$ \\
\hline $4 d$ & $65,50 \pm 12,11 a$ & $48,61 \pm 15,67 b, y$ & $67,90 \pm 19,39 a, y z$ & $31,54 \pm 8,60 c, y$ & $* * *$ \\
\hline $8 d$ & $74,30 \pm 12,78 a$ & $73,21 \pm 19,16 a, x$ & $80,49 \pm 11,04 a, x y$ & $38,27 \pm 12,24 b, x y$ & $* * *$ \\
\hline $12 \mathrm{~d}$ & $75,61 \pm 16,43 a$ & $71,33 \pm 11,67 a, x$ & $81,44 \pm 14,02 a, x$ & $43,63 \pm 10,24 b, x$ & $* * *$ \\
\hline Sig. & ns & $* * *$ & $* * *$ & * & \\
\hline
\end{tabular}

${ }^{1}$ BL: Bermeya ligera, BP: Bermeya pesada, MG: Murciano-Granadina (lechal, ligera), TA: Ternasco de Aragón IGP (ovino).

a, b: indican diferencias en una fila $(P<0,05)$.

$\mathrm{x}, \mathrm{y}$ : indican diferencias en una columna $(P<0,05)$.

los dos productos de referencia (MG y TA). Igualmente se observa que dichos valores experimentan un inesperable y no fácil de explicar, incremento a lo largo de la maduración, especialmente entre los días 4 y 8, lo que podría ser debido a alteraciones de la estructura muscular con incremento de la densidad del tejido conjuntivo en la muestra a analizar. La mayoría de las comparaciones entre razas y especies están basadas en datos obtenidos en la fuerza de corte/cizallamiento, donde estos dos componentes previamente descritos interactúan juntos, siendo los resultados de la bibliografía poco homogéneos. Autores como Sen et al. (2004) describieron que la carne de ovino es más tierna que la de procedente de la especie caprina, asociando estas diferencias a la variación en el contenido en colágeno, grasa y composición de la fibra muscular entre especies. En cambio, otros como Santos et al. (2008) obtuvieron resultados contrarios, siendo mayor la fuerza de corte necesaria para ovinos que para caprinos de la misma precocidad, asociando este hecho a las diferencias en tamaño de la canal, cantidad de grasa subcutánea y la velocidad de enfriamiento de la canal. En relación a los resultados obtenidos en el presente estudio se observa como la maduración produjo más variación en las características de la carne de los tipos de referencia que en la de los animales bermeyos. 
Ácidos grasos

En la Tabla 4 se muestra la composición en ácidos grasos del músculo, expresados como \% del total de ácidos grasos, siendo los predominantes en la especie caprina C16:0, C18:0 y C18:1 n-9 como refleja éste y otros trabajos (Dhanda et al., 2003; Horcada et al., 2012; 2014). Se observan, en general, diferencias importantes entre los tipos comerciales comparados en casi todos los ácidos grasos analizados, excepto C20:2 n-6, C20:3 n-6 y C22:0. De esta manera, ácidos grasos relacionados directamente (C12:0) o indirectamente (C14:0) con la ingesta de leche aparecen en mayor proporción en aquellos animales más jóvenes que están tomando una dieta láctea. A pesar de no haber diferencias entre los cabritos de raza Bermeya entre sí en el porcentaje de $\mathrm{C} 16: 0$, los porcentajes fueron menores que en los otros dos tipos estudiados $(P<0,01)$. Sí se observaron diferencias en el porcentaje de C18:0, donde el Bermeyo pesado tuvo valores superiores al resto de animales $(P<0,001)$, quizás como consecuencia de su mayor edad de sacrificio. Sin embargo, no se mostraron diferencias en los niveles totales de ácidos grasos saturados entre los tipos estudiados. De acuerdo con las comparaciones entre diversas razas caprinas realizadas por Horcada et al. $(2012,2014)$, el perfil lipídico de razas lecheras es más similar al perfil que presentan los ovinos que al de las razas caprinas de aptitud cárnica, resultado que concuerda en un importante número de ácidos grasos hallados en el presente estudio.

Por contra, aparecieron diferencias en el porcentaje de ácidos grasos monoinsaturados $(P$ $<0,001)$, presentado los cabritos bermeyos de ambos pesos niveles claramente inferiores al cabrito Murciano-Granadino y, especialmente, al Ternasco de Aragón, debido fundamentalmente a su menor porcentaje de C18:1 n-9. La alimentación que reciben los animales (y las madres) podría haber afectado a este menor contenido en C18:1 n-9. No obstante, los cabritos de raza Bermeya presentaron un mayor porcentaje de ácidos grasos poliinsaturados, especialmente los de peso ligero $(P<$ $0,001)$, por el mayor porcentaje de ácidos grasos $n-6$, debido al mayor contenido en C18:2 $n-6$ y C20:4 $n-6$. También se observa un mayor porcentaje de ácidos grasos $n-3(P<0,001)$ en los cabritos bermeyos, mayor en el ligero que en el pesado, pero significativamente superiores al resto de animales estudiados. Esto podría ser reflejo del pastoreo de estos animales y/o de sus madres, lo que provocó una mayor incorporación de C18:3n-3, característico del forraje (Valvo et al., 2003), a diferencia de los resultados hallados en la raza Murciano-Granadino y el Ternasco de Aragón, cuya alimentación se basa, fundamentalmente, en cereales. Esto produce un cociente poliinsaturados/saturados mayor y más deseable en los animales de raza Bermeya ligeros $(P<0,001)$, y un menor ratio $n-6 / n-3$ en ambos pesos de la raza Bermeya.

\section{Calidad sensorial}

En la Tabla 5 se recogen los resultados obtenidos por medio del panel entrenado. Hubo diferencias en la intensidad de olor a especie $(P<0,05)$, terneza, jugosidad e intensidad de flavor a especie $(P<0,001)$ entre los cuatro tipos de animales estudiados. En relación a la raza Bermeya, el único atributo que presentó valores significativamente diferentes entre los dos tipos de animales (ya sean ligeros o pesados) fue la terneza, parámetro en el cual los bermeyos pesados obtuvieron los valores más bajos, probablemente debido a su mayor edad o al sistema de producción extensivo, presentando la carne ovina los valores más altos de terneza (Guerrero et al., 2014). Los demás atributos fueron similares entre los diferentes tipos de animales. Estos resultados coinciden con los datos de textura en la compresión al $80 \%$, a pesar de que la compresión 
Tabla 4. Composición en ácidos grasos (\%) de la grasa intramuscular del músculo Longissimus thoracis en cabritos comerciales de raza Bermeya sacrificados a dos pesos, respecto a dos tipos comerciales de referencia ${ }^{1}$

Table 4. Fatty acid composition (\%) from intramuscular fat of Longissimus thoracis muscle of Bermeya commercial kids slaughtered at two different weights compared and compared with two commercial small ruminant types

\begin{tabular}{|c|c|c|c|c|c|}
\hline & $B L$ & $\mathrm{BP}$ & MG & TA & \\
\hline C10:0 & $0,27 \pm 0,16$ & $0,19 \pm 0,11$ & $0,26 \pm 0,11$ & $0,17 \pm 0,06$ & $\mathrm{t}$ \\
\hline C12:0 & $0,48 \pm 0,33 a b$ & $0,24 \pm 0,15 c$ & $0,65 \pm 0,23 a$ & $0,28 \pm 0,20 \mathrm{bc}$ & $* * *$ \\
\hline C14:0 & $4,54 \pm 1,63 b$ & $3,85 \pm 2,00 b$ & $5,95 \pm 1,48 a$ & $3,30 \pm 1,49 b$ & ** \\
\hline C14:1 & $0,09 \pm 0,08 b$ & $0,06 \pm 0,05 b$ & $0,18 \pm 0,04 a$ & $0,10 \pm 0,07 b$ & $* * *$ \\
\hline C15:0 & $0,53 \pm 0,22$ & $0,48 \pm 0,17$ & $0,35 \pm 0,10$ & $0,46 \pm 0,12$ & $\mathrm{t}$ \\
\hline C16:0 & $20,42 \pm 5,12 b c$ & $19,15 \pm 3,31 \mathrm{c}$ & $23,92 \pm 2,38 a$ & $22,58 \pm 1,99 a b$ & ** \\
\hline C16:1 & $1,28 \pm 0,98 a b$ & $0,85 \pm 0,34 b$ & $1,59 \pm 0,22 a$ & $1,45 \pm 0,30 a$ & * \\
\hline C17:0 & $0,94 \pm 0,25 c$ & $1,23 \pm 0,23 b$ & $0,70 \pm 0,09 d$ & $1,58 \pm 0,15 a$ & $* * *$ \\
\hline C17:1 & $0,40 \pm 0,14 b$ & $0,46 \pm 0,17 b$ & $0,42 \pm 0,09 b$ & $0,77 \pm 0,21 a$ & $* * *$ \\
\hline C18:0 & $14,66 \pm 2,51 b$ & $21,02 \pm 5,24 a$ & $13,94 \pm 1,38 b$ & $15,65 \pm 2,90 b$ & *** \\
\hline tC18:1 n-9 & $1,44 \pm 0,72 c$ & $2,51 \pm 0,84 b$ & $1,48 \pm 0,60 c$ & $4,53 \pm 1,41 a$ & $* * *$ \\
\hline C18:1 n-9 & $23,29 \pm 7,60 \mathrm{c}$ & $27,56 \pm 5,68 b$ & $35,75 \pm 2,61 a$ & $34,51 \pm 2,30 a$ & *** \\
\hline C18:1 n-7 & $1,11 \pm 0,28 a b$ & $0,86 \pm 0,41 b$ & $0,95 \pm 0,23 b$ & $1,34 \pm 0,45 a$ & * \\
\hline tC18:2 n-6 & $0,22 \pm 0,13 b$ & $0,24 \pm 0,13 b$ & $0,36 \pm 0,06 a$ & $0,09 \pm 0,08 c$ & $* * *$ \\
\hline C18:2 n-6 & $8,57 \pm 3,91 a$ & $5,90 \pm 2,55 b$ & $5,51 \pm 1,75 b$ & $5,44 \pm 1,29 b$ & * \\
\hline $\mathrm{CLA}^{2}$ & $0,63 \pm 0,24$ & $0,68 \pm 0,21$ & $0,72 \pm 0,18$ & $0,50 \pm 0,25$ & $\mathrm{t}$ \\
\hline C18:3 n-6 & $0,06 \pm 0,04 a b$ & $0,04 \pm 0,04 b$ & $0,06 \pm 0,03 a b$ & $0,08 \pm 0,03 a$ & $\mathrm{t}$ \\
\hline C18:3n-3 & $1,63 \pm 0,56 a$ & $1,90 \pm 0,61 a$ & $0,27 \pm 0,06 b$ & $0,49 \pm 0,41 b$ & $* * *$ \\
\hline C20:0 & $0,17 \pm 0,05 a$ & $0,19 \pm 0,05 a$ & $0,10 \pm 0,03 b$ & $0,13 \pm 0,03 b$ & $* * *$ \\
\hline C20:1 & $0,11 \pm 0,02 a b$ & $0,10 \pm 0,02 b$ & $0,13 \pm 0,01 a$ & $0,12 \pm 0,02 a$ & ** \\
\hline C20:2 n-6 & $0,08 \pm 0,05$ & $0,15 \pm 0,28$ & $0,03 \pm 0,03$ & $0,06 \pm 0,02$ & ns \\
\hline$C 20: 2 n-3$ & $0,41 \pm 0,25 a$ & $0,23 \pm 0,11 b$ & $0,15 \pm 0,08 b$ & $0,14 \pm 0,04 b$ & $* * *$ \\
\hline C20:3 n-6 & $0,06 \pm 0,09$ & $0,06 \pm 0,05$ & $0,07 \pm 0,05$ & $0,07 \pm 0,06$ & ns \\
\hline C20:4 n-6 & $4,98 \pm 3,09 a$ & $2,46 \pm 1,28 b$ & $2,27 \pm 1,15 b$ & $1,67 \pm 0,63 b$ & *** \\
\hline C20:5 n-3 & $2,13 \pm 1,33 a$ & $1,00 \pm 0,45 b$ & $0,16 \pm 0,07 c$ & $0,19 \pm 0,11 \mathrm{c}$ & $* * *$ \\
\hline C22:0 & $0,28 \pm 0,17$ & $0,17 \pm 0,09$ & $0,26 \pm 0,15$ & $0,20 \pm 0,08$ & ns \\
\hline$C 22: 5 n-3$ & $2,13 \pm 1,27 a$ & $1,21 \pm 0,55 b$ & $0,49 \pm 0,16 c$ & $0,39 \pm 0,16 c$ & *** \\
\hline C22:6 n-3 & $0,60 \pm 0,31 a$ & $0,29 \pm 0,11 b$ & $0,12 \pm 0,05 c$ & $0,13 \pm 0,05 c$ & $* * *$ \\
\hline $\mathrm{AGS}^{3}$ & $42,30 \pm 8,96$ & $46,51 \pm 7,04$ & $46,13 \pm 4,13$ & $44,35 \pm 3,39$ & ns \\
\hline $\mathrm{AGMI}^{4}$ & $27,73 \pm 8,40 \mathrm{~b}$ & $32,40 \pm 6,70 b$ & $40,49 \pm 2,68 a$ & $42,82 \pm 3,68 a$ & $* * *$ \\
\hline $\mathrm{AGPI}^{5}$ & $21,49 \pm 10,19 a$ & $14,16 \pm 4,48 b$ & $10,23 \pm 3,27 b$ & $9,24 \pm 1,67 b$ & $* * *$ \\
\hline$n-6$ & $14,31 \pm 7,15 a$ & $9,03 \pm 3,72 b$ & $8,39 \pm 2,94 b$ & $7,47 \pm 1,72 b$ & $* *$ \\
\hline$n-3$ & $6,54 \pm 3,30 a$ & $4,46 \pm 1,38 b$ & $1,12 \pm 0,36 \mathrm{c}$ & $1,28 \pm 0,64 c$ & $* * *$ \\
\hline AGPI/AGS & $0,58 \pm 0,38 a$ & $0,32 \pm 0,15 b$ & $0,23 \pm 0,10 b$ & $0,21 \pm 0,05 b$ & $* * *$ \\
\hline$n-6 / n-3$ & $2,22 \pm 0,35 b$ & $2,26 \pm 1,56 b$ & $7,79 \pm 1,94 a$ & $7,01 \pm 2,74 a$ & $* * *$ \\
\hline
\end{tabular}

${ }^{1}$ BL: Bermeya ligera, BP: Bermeya pesada, MG: Murciano-granadina (lechal, ligera), TA: Ternasco de Aragón IGP (ovino). $\mathrm{t}=P<0,10$.

2 Ácido linoleico conjugado; ${ }^{3}$ Ácidos grasos saturados; ${ }^{4}$ Ácidos grasos monoinsaturados; ${ }^{5}$ Ácidos grasos poliinsaturados.

Distintas letras en una fila indican diferencias $(P<0,05)$. 
Tabla 5. Calidad sensorial del músculo Longissimus lumborum evaluadas por un panel entrenado en cabritos comerciales de raza Bermeya sacrificados a dos pesos, respecto a dos tipos comerciales de referencia ${ }^{1}$

Table 5. Sensory quality of the Longissimus lumborum muscle evaluated by a trained panel from Bermeya commercial kids slaughtered at two different weights and compared with two commercial small ruminant types

\begin{tabular}{|c|c|c|c|c|c|}
\hline & $\mathrm{BL}$ & BP & MG & TA & \\
\hline \multicolumn{6}{|c|}{ Intensidad de olor ${ }^{2}$} \\
\hline Especie & $4,52 \pm 0,85 b$ & $4,90 \pm 0,74 a b$ & $5,29 \pm 0,44 a$ & $5,54 \pm 0,89 a$ & * \\
\hline Grasa & $3,35 \pm 0,83$ & $2,91 \pm 1,0$ & $3,54 \pm 0,69$ & $3,34 \pm 0,79$ & ns \\
\hline \multicolumn{6}{|l|}{ Textura ${ }^{2}$} \\
\hline Terneza & $5,94 \pm 1,00 a$ & $4,66 \pm 0,85 b$ & $5,87 \pm 1,46 a$ & $6,33 \pm 1,15 a$ & $* * *$ \\
\hline Jugosidad & $4,98 \pm 0,56 a b$ & $4,46 \pm 0,44 b$ & $4,73 \pm 0,53 a b$ & $5,15 \pm 0,66 a$ & $* * *$ \\
\hline Fibrosidad & $4,0 \pm 1,18$ & $5,04 \pm 0,93$ & $4,33 \pm 1,0$ & $3,94 \pm 0,94$ & ns \\
\hline Untuosidad & $3,90 \pm 0,76$ & $3,47 \pm 0,50$ & $3,91 \pm 0,92$ & $3,93 \pm 0,77$ & ns \\
\hline \multicolumn{6}{|c|}{ Intensidad de flavor ${ }^{2}$} \\
\hline Especie & $4,81 \pm 0,79 b$ & $4,78 \pm 0,58 b$ & $5,25 \pm 0,55 a b$ & $5,75 \pm 0,50 a$ & $* * *$ \\
\hline Metálico & $3,04 \pm 0,95$ & $2,80 \pm 0,67$ & $2,77 \pm 0,56$ & $2,68 \pm 0,70$ & ns \\
\hline Grasa & $4,43 \pm 0,55$ & $3,83 \pm 0,72$ & $4,43 \pm 0,75$ & $4,45 \pm 0,71$ & ns \\
\hline Lácteo & $1,61 \pm 0,59$ & $1,30 \pm 0,57$ & $1,85 \pm 0,74$ & $1,93 \pm 0,50$ & ns \\
\hline Ácido & $2,56 \pm 0,73$ & $2,56 \pm 0,66$ & $2,83 \pm 0,33$ & $2,74 \pm 0,61$ & ns \\
\hline Picante & $2,02 \pm 0,69$ & $1,66 \pm 0,75$ & $2,13 \pm 0,79$ & $2,15 \pm 0,45$ & ns \\
\hline
\end{tabular}

${ }^{1}$ BL: Bermeya ligera, BP: Bermeya pesada, MG: Murciano-granadina (lechal, ligera), TA: Ternasco de Aragón IGP (ovino).

2 Escala 1-10.

Distintas letras en una fila indican diferencias $(P<0,05)$.

se realiza sobre carne cruda, y el cocinado produce cambios drásticos en las características del producto (Campo et al., 2000).

En relación a la intensidad de olor y flavor a especie, éstos fueron superiores en la raza Murciano-Granadina $y$, especialmente, en el Ternasco de Aragón $(P<0,05$ y $P<0,001$, respectivamente para intensidad de olor y flavor), hecho asociado a la mayor cantidad de grasa, lo cual conlleva una mayor concen- tración de compuestos volátiles responsables de la producción de dichos aromas (Horcada et al., 2014).

\section{Consumidores}

Se observa como la aceptabilidad global y de la terneza varió entre los cuatro tipos de animales $(P<0,001)$ (Tabla 6), no encontrándose diferencias en la aceptabilidad del sabor. Los 
Tabla 6. Aceptabilidad por parte de los consumidores $(n=100)$ de la carne de cabritos de raza Bermeya sacrificados a dos pesos, respecto a dos tipos comerciales de referencia ${ }^{1}$

Table 6. Consumer meat acceptability of Bermeya kids slaughtered at two different weights compared with and compared with two commercial small ruminant types $(n=100)$

\begin{tabular}{lccccc}
\hline Atributo & BL & BP & MG & TA & Sig. \\
\hline Aceptabilidad de Terneza $^{2}$ & $6,74 \pm 1,66$ b & $5,95 \pm 1,96 \mathrm{c}$ & $7,55 \pm 1,31$ a & $7,49 \pm 1,33$ a & $* * *$ \\
Aceptabilidad de Sabor $^{2}$ & $6,65 \pm 1,71$ & $6,52 \pm 1,66$ & $7,03 \pm 1,60$ & $6,64 \pm 1,95$ & ns \\
Aceptabilidad Global $^{2}$ & $6,65 \pm 1,75$ b & $6,15 \pm 1,79$ b & $7,15 \pm 1,51$ a & $6,96 \pm 1,78$ a & $* * *$ \\
\hline
\end{tabular}

${ }^{1}$ BL: Bermeya ligera, BP: Bermeya pesada, MG: Murciano-granadina (lechal, ligera), TA: Ternasco de Aragón IGP (ovino).

${ }^{2}$ Escala de aceptación ( 1 = me desagrada extremadamente; 9 = me gusta extremadamente).

Distintas letras en la misma fila indican diferencias $(P<0,05)$.

animales de referencia (MG y TA) obtuvieron una mayor puntuación, mostrando los lechales bermeyos ligeros una puntuación de aceptabilidad de la terneza intermedia concordando parcialmente por lo descrito por el panel entrenado. La aceptabilidad global del tipo bermeyo pesado fue inferior a los animales de referencia, sin embargo no difiere estadísticamente con el tipo lechal. Estos resultados indicarían que el consumidor no percibe el producto como diferente, a pesar de proceder de pesos, edades y sistemas de producción diferentes.

Los datos obtenidos en este trabajo concuerdan con los resultados de los estudios de consumidores realizados por Guerrero et al. (2014), donde se estudiaron 5 razas caprinas de distintas aptitudes y una ovina. Al igual que en el actual trabajo, los animales sacrificados a corta edad y procedentes de razas lecheras (Murciano-Granadina) fueron los mejor valorados por el consumidor español, estando la aceptabilidad global de la carne en pequeños rumiantes fuertemente correlacionada con el flavor y la terneza (Guerrero et al., 2014). En este estudio los consumidores no encontraron diferencias en la aceptabilidad del sabor, siendo por tanto la textura (terneza) el atributo que posiblemente más influyó en la definición de la aceptabilidad global.

En general, la aceptabilidad de los diferentes grupos de animales estudiados y para todos los parámetros analizados, se podría considerar como buena, obteniéndose valoraciones superiores al 5,5 sobre una escala hedónica de 9 puntos.

\section{Conclusiones}

Los dos tipos de productos procedentes de la raza caprina Bermeya (ligeros y pesados) difieren en las características de calidad de la canal, lo que estaría asociado a las diferencias en peso y edad de sacrificio entre los dos tipos de animales. Igualmente, los animales del tipo pesado de la raza Bermeya presentaron una carne con mayor porcentaje de proteína, más roja, con mayor croma y una menor proporción de ácidos grasos poliinsaturados que los del tipo de raza Bermeya ligero. Sin embargo, a nivel sensorial, ambos presentaron una aceptación semejante, aun siendo los lechales ligeros más tiernos que los animales pesados. 
Los resultados obtenidos en los parámetros de calidad estudiados de la raza Bermeya, incluyendo la aceptación del producto por parte de los consumidores, están dentro de unos rangos aceptables y comparables con las de dos productos de referencia utilizados en el presente trabajo, lo que reflejaría su potencial para la producción de "carne de calidad" y daría unas buenas expectativas en cuanto a las posibilidades futuras de su conservación.

\section{Agradecimientos}

ACRIBER, Gobierno del Principado de Asturias, al panel de consumidores y demás participantes en el proyecto, así como al personal del Departamento que colaboró en este trabajo. V.C. Resconi está financiada por un Contrato del Programa Juann de la Ciervam (Ministerio de Economía y Competitividad), España.

\section{Bibliografía}

Álvarez A (2008). La cabra Bermeya. Ed. ACRIBER y Gobierno del Principado de Asturias. 46 pp.

BOE (2009). Real Decreto 2129/2008, de 26 de diciembre, por el que se establece el Programa nacional de conservación, mejora y fomento de las razas ganaderas. Boletín Oficial del Estado $N^{\circ} 23$, Sec. I, 9211-9242.

Bonvillani A, Peña F, Gea G, Gómez G, Petryna A, Perea J (2010). Carcass characteristics of Criollo Cordobés kid goats under an extensive management system: Effects of gender and liveweight at slaughter. Meat Science 86: 651-659.

Bligh EG, Dyer WJ (1959). A rapid method of total lipid extraction and purification. Canadian Journal Biochemistry and Physiology 37: 911-917.

Campo MM, Santolaria P, Sañudo C, Lepetit J, Olleta JL, Panea B, Alberti P (2000). Assessment of breed type and ageing time effects on beef meat quality using two different texture devices. Meat Science 55: 371-378.
Carrillho MC, López M, Campo MM (2009). Effect of the fattening diet on the development of the fatty acid profile in rabbits from weaning. Meat Science 83: 85-95.

Colomer F, Morand Fehr p, Kirton AH, Delfa R, Sierra I (1988). Métodos normalizados para el estudio de los caracteres cuantitativos y cualitativos de las canales caprina y ovinas. Cuadernos INIA n 17, 41 pp.

Dhanda JS, Taylor DG, Murray PJ (2003). Part2. Carcass composition and fatty acid profiles of adipose tissue of male goats: effect of genotype and liveweight at slaughter. Small Ruminant Research 50: 67-74.

Esteban C (2008). Bermeya. En: Razas ganaderas españolas caprinas. Ed.: FEAGAS, Madrid, pp. 91-104.

Figueroa P, Fernández I, Gómez E, Royo LJ, Álvarez I, Goyache F (2003). Iniciativas de conservación de la cabra de raza Bermeya de Asturias. Animal Genetic Resources Information 33: 57-71.

Guerrero A, Campo MM, Cilla I, Olleta JL, Alcalde MJ, Horcada A, Sañudo C (2014). A comparison of laboratory-based and home-based tests of consumer preferences using goat and lamb meat. Journal of Sensory Studies 29: 201-210.

ISO (1973). Meat and meat products - Determination of total fat content. Method ISO R-1442. International Organization for Standardization. Ginebra, Suiza.

ISO (1978). Meat and meat products - Determination of nitrogen content. Method ISO R-937. International Organization for Standardization. Ginebra, Suiza.

ISO (1997). Meat and meat products - Determination of moisture content. Method ISO R1442. International Organization for Standardization. Ginebra, Suiza.

ISO (1998). Meat and meat products - Determination of total ash content. Method ISO R-936. International Organization for Standardization. Ginebra, Suiza.

García R, Celaya R, García U, Osoro K (2012). Goat grazing, its interactions with other herbivores and biodiversity conservation issues. Small Ruminant Research 107: 49-64. 
Horcada A, Ripoll G, Alcalde MJ, Sañudo C, Teixeira A, Panea B (2012). Fatty acid profile of three adipose depots in seven Spanish breeds of suckling kids. Meat Science 92: 89-96.

Horcada A, Campo MM, Polvillo O, Alcalde MJ, CiIla I, Sañudo C (2014). A comparative study of fatty acid profiles of fat in commercial Spanish suckling kid and lambs. Spanish Journal Agricultural Research 12: 427-435.

Lepetit J, Culioli J (1994). Mechanical properties of meat. Meat Science 36: 203-237.

Macfie HJ, Bratchell N, Greenhoff K, Vallis LV (1989). Designs to balance the effect of order presentation and first-order and carry over effects in hall test. Journal of Sensory Studies 4: 129-148.

MAGRAMA (2014). Indicación Geográfica protegida Ternasco de Aragón. Pliego de condiciones. Disponible en www.magrama.gob.es/es/alimentacion/temas/calidad-agroalimentaria/calid ad-diferenciada/dop/carnes/dop_ternasco_aragon.aspx. (24 Noviembre 2014).

Martínez-Cerezo S, Sañudo C, Panea B, Medel I, Delfa R, Sierra I, Beltrán JA, Cepero R, Olleta JL (2005). Breed, slaughter weight and ageing time effects on physico-chemical characteristics of lamb meat. Meat Science 69: 325-333.

UNE-EN ISO 8586-2. 2009. Análisis sensorial. Guía general para la selección, entrenamiento y control de evaluadores.

Santos VAC, Silva SR, Azevedo JMT (2008). Carcass composition and meat quality of equally mature kids and lambs. Journal Animal Science 86: 1943-1950.

Sañudo C, Sierra I, Alcalde MJ, Rota A, Osorio JC (1993). Calidad de la canal y de la carne en corderos ligeros y semipesados de las razas Rasa Aragonesa, Lacaune y Merino Alemán. Información Técnica Económica Agraria 89: 230-214.
Sañudo C, Campo MM, Sierra I, María G, Olleta JL, Santolaria P (1997). Breed effect on carcass and meat quality of suckling lambs. Meat Science 46: 357-365.

Sañudo C (2008). Calidad de la canal y de la carne ovina y caprina y los gustos de los consumidores. Revista Brasileira de Zootecnia 37: 143-160.

Sañudo C, Campo MM, Muela E, Olleta JL, Delfa R, Jiménez-Badillo R, Alcalde MJ, Horcada A, Oliveira I, Cilla I (2012). Carcass characteristics and instrumental meat quality of suckling kids and lambs. Spanish Journal of Agricultural Research 10: 690-700.

Sen AR, Santra A, Karim SA (2004). Carcass yield, composition and meat quality attributes of sheep and goat under semiarid conditions. Meat Science 66: 757-763.

Valvo MA, Lanza M, Bella M, Fasone V, Scerra M, Biondi L, Priolo A (2003). Effect of ewe feeding system (grass v. concentrate) on intramuscular fatty acids of lambs raised exclusively on maternal milk. Animal Science 81: 431-436.

Vergara H (2005). Composición regional y tisular de la canal ovina. En: Estandarización de las metodologías para evaluar la calidad del producto (animal vivo, canal, carne y grasa) en los rumiantes (Coord. V Cañeque, C Sañudo), pp. 170-188. Monografías, Serie Ganadera 3, INIA, Madrid, España.

Zurita-Herrera P, Delgado JV, Argüelo A, Camacho ME (2011). Multivariate analysis of meat production traits in Murciano-Granadina goat kids. Meat Science 88: 447-453.

(Aceptado para publicación el 28 de julio de 2015) 